\title{
Corps et culture
}

Numéro 2 | 1997

Plaisirs du corps, plaisirs du sport

\section{Plaisir et handicap physique}

Contribution à une approche scientifique du plaisir

Jean-Philippe Turpin, Jean-Marc Barbin, Gilles Bui-Xuân, Anne Marcellini, Jacques Mikulovic, Grégory Ninot et Guy Haye

\section{CpenEdition}

Journals

Édition électronique

URL : http://journals.openedition.org/corpsetculture/389

DOI : 10.4000/corpsetculture.389

ISSN : $1777-5337$

Éditeur

Association Corps et Culture

Édition imprimée

Date de publication : 1 juin 1997

ISSN : 1268-5631

Référence électronique

Jean-Philippe Turpin, Jean-Marc Barbin, Gilles Bui-Xuân, Anne Marcellini, Jacques Mikulovic, Grégory Ninot et Guy Haye, "Plaisir et handicap physique », Corps et culture [En ligne], Numéro 2 | 1997, mis en ligne le 18 juin 2007, consulté le 08 septembre 2020. URL : http://journals.openedition.org/ corpsetculture/389; DOI : https://doi.org/10.4000/corpsetculture.389

Ce document a été généré automatiquement le 8 septembre 2020

(c) tous droits réservés 


\title{
Plaisir et handicap physique
}

\author{
Contribution à une approche scientifique du plaisir \\ Jean-Philippe Turpin, Jean-Marc Barbin, Gilles Bui-Xuân, Anne Marcellini, \\ Jacques Mikulovic, Grégory Ninot et Guy Haye
}

1 Suite à une proposition de recherche sur la thématique des plaisirs du corps et des plaisirs du sport, un regroupement original s'est construit autour du thème «Personnes handicapées physiques et plaisir ». Professionnels en Activités

Physiques et Sportives auprès de personnes handicapées, chercheurs travaillant sur le thème du handicap depuis de nombreuses années, spécialistes de la question du plaisir sont autant de compétences qui se sont mises en commun l'espace d'une étude. C'est en pariant sur cette diversité que s'est construit ce travail.

Etudier le plaisir à partir du handicap ?Plaisir et handicap ?

3 Poser la question du plaisir, en relation avec le handicap, n'est pas simple : c'est un thème rarement abordé, et sur lequel peu de travaux sont disponibles. Deux raisons essentielles semblent pouvoir expliquer cet état de fait. D'une part, la difficulté de l'approche intersubjective de la question du plaisir, en particulier dans ses développements relatifs à la sexualité et à l'intimité du sujet, peut générer une gêne dans l'interaction chercheur-sujet. D'autre part, la thématique du plaisir présentée à des personnes considérées comme en souffrance peut sembler déplacée.

4 Notre expérience professionnelle d'intervenants en Activités Physiques Adaptées auprès de personnes handicapées physiques nous met en situation quotidienne d'observation de manifestations verbales attestant de l'accès de ces personnes au plaisir, Si aucun doute ne subsiste alors quant à l'existence de ce plaisir, nous nous sommes interrogés sur les processus et les modalités que ces personnes déploient pour y accéder.

Une définition du plaisir?

5 l'out indique que le plaisir est un « ressenti », un « éprouvé » homogène, de l'ordre de l'évidence mais dont les causes sont multiformes. Sans chercher à définir a priori cette subjectivité familière, nous nous sommes interrogés sur les associations faites par les 
personnes handicapées à la notion de plaisir en général, et des plaisirs du corps et du sport en particulier.

Notre démarche

6 La démarche est exploratoire, et a pour but de tenter d'appréhender un public particulier dans ses relations avec le plaisir, ceci dans une double perspective : accéder à une meilleure connaissance des personnes handicapées

7 Une première question a animé le groupe : existe-t-il des plaisirs spécifiques chez les personnes handicapées physiques? Répondre à cette question supposait une réflexion sur la méthode à retenir pour aborder ce thème d'un point de vue scientifique.

MéthodeOutil de recueil des données

8 Le plaisir est, dans tous les cas, malgré l'ignorance que nous avons des processus qui le génèrent, une impression, une sensation, conscientes, qui peuvent être verbalisées. Par conséquent, toutes les approches à partir du discours sont pertinentes. Mais elles ne sont pas suffisantes car on connait bien les pièges des rationalisations et des actes verbaux défensifs.

9 Parce que le plaisir aboutit à des phénomènes de répétition (le plaisir est toujours recherché), toutes les techniques d'observation sont en mesure d'apporter des informations.

S'appuyer sur les discours et sur les observations sont donc des propositions méthodologiques à retenir pour une approche scientifique du plaisir.

11 Dans ce travail, nous avons fait le choix de l'exploration par entretiens cliniques (de type semi-directif), et décidé d'aborder d'emblée la question du plaisir en étant particulièrement attentifs aux réactions des personnes interviewées: refus, étonnement, agressivité, etc.

Population d'étude

12 Notre population d'étude a été constituée à partir d'un critère de diversité : nature du handicap (de naissance ou traumatique), degré du déficit (marche appareillée, fauteuil roulant manuel ou électrique), ancienneté dans le handicap, sexe, sportifs et nonsportifs.

13 Elle regroupe 8 personnes de 18 à 47 ans, recrutées de façon aléatoire.

Analyse des discours

14 Pour l'analyse des entretiens, une première lecture flottante a été menée, sous la forme d'une analyse thématique simple, pour dégager des thèmes généraux relatifs au plaisir. A travers cette lecture nous avons testé l'hypothèse selon laquelle les personnes handicapées éprouvaient deux types de plaisir : des plaisirs "ordinaires ", c'est-à-dire équivalents à ceux que peut éprouver toute personne valide, et des plaisirs "spécifiques".

15 La validation de cette hypothèse pourrait nous amener à d'autres lectures des entretiens visant à mieux comprendre ces plaisirs " particuliers ».

Premiere lecture des entretiens : quand les handicapés prennent du plaisir...Spécificités des plaisirs ou plaisirs communs?

16 La récapitulation des thèmes repérés fait apparaitre deux types de discours: un discours que nous percevons comme « ordinaire, dans le sens où nous y retrouvons des associations communes du type plaisir de lire, d'écouter de la musique, de faire un bon repas, de voir des amis, etc., et un discours « déroutant » par l'apparition de thèmes qui 
nous surprennent dans leur association au plaisir. Un discours qui nous place dans un rapport de proximité avec les sujets interviewés, quand un autre marque leur différence par rapport à nous.

Des plaisirs « ordinaires"

«Il y a le plaisir de la douche, là je me régale, prendre une bonne douche chaude. Je peux rester des heures en faisant couler l'eau sur moi, et je fais rien, je me régale.

."

«J'adore manger aussi, la bonne bouffe j'adore ça, le bon vin... ».

«Il y a aussi le plaisir intellectuel je dirais... Regarder un bon film, un film

exceptionnel... Il n'y a pas de sensation physique, c'est le cerveau qui travaille » (A)

La lecture d'extraits de discours qui nous sont apparus, à nous valides, comme " étranges ", mérite réflexion. Ces situations présentées comme pourvoyeuses de plaisir mettent en scène la personne handicapée dans un rapport au social "commun", " quotidien ». On ne peut pas dire qu'on ne comprend pas le plaisir ressenti lors de ces situations, mais nous ne l'éprouvons pas, ou plus exactement nous ne l'éprouvons plus. Elles évoquent pour nous, adultes valides, des «obligations", voire des contraintes, et nous pouvons même les percevoir comme sources de déplaisir. Pour les personnes handicapées, il semble bien que ce soit la banalité, la quotidienneté même de ces situations qui les leur rendent plaisantes. Comme si leur situation d'exclusion ou d'exception donnait un goût très particulier à toute participation sociale « banale ».

Des plaisirs « étranges »

«Le plaisir de sortir, oui sortir seule de la maison, aller en ville, ou aller faire des courses au supermarché du coin, c'est un plaisir. » (B)

«Il y a le plaisir de se dire bon, on s'habille parce qu'on va chez le kiné ou acheter une baguette de pain... ». (E)

« Vous voyez cette dame qui était là tout à l'heure, c'est la première fois qu'on s'est vu hein, elle m'a dit : «Vous acceptez de prendre un pot? J'ai dit : écoutez je suis désolée j'attends quelqu'un et j'ai peur de le rater et on s'est donné rendez-vous ici, » et elle m'a dit «ce sera pour une autrefois... » Mais c'est un plaisir immense !. (E)

18 Par exemple sont présentés comme sources de plaisir le fait d'aller faire les courses au supermarché, de rouler en voiture, de sortir seul de la maison, d'être dans des endroits où il $\mathrm{y}$ a beaucoup de monde, au milieu des autres. On note ici le plaisir associé plus directement au dépassement d'une certaine immobilité physique, de la dépendance, de l'isolement social.

On peut donc bien repérer chez les personnes handicapées physiques des plaisirs " ordinaires" et des plaisirs "spécifiques». Ces derniers sont marques par un axe dominant qu'une des personnes a bien résumé : «le plaisir de gagner de la validité ». On peut lire cette expression au premier degré: ils ont du plaisir lorsqu'ils se rapprochent du mode de vie de ceux qu'ils appellent les valides, c'est-à-dire nous. Mais l'expression recèle peut-être aussi une dynamique d'affirmation de soi comme valide au sens de « valable ».

Finalement, ce type de discours renvoie principalement à des plaisirs qui sont référés à un dépassement de limites, propres aux personnes interviewées. Mais si ce discours présente une certaine étrangeté, il ne nous est pas pour autant étranger: il nous rappelle en effet des discours adolescents, par la récurrence de l'expression des plaisirs de l'émancipation et de l'autonomisation.

Des plaisirs spécifiques et une problématique « adolescente »? 
21 Pourquoi retrouve-t-on chez les personnes handicapées cette problématique de l'adolescence? De nombreux travaux mettent en évidence la crise identitaire consécutive à une telle paraplégie ou de la tétraplégie, (Bon C., 1995). Il semble que leur problématique ceux qui ont toujours vécu avec leur handicap, ne soit pas très lointaine de celle des handicapés d'origine traumatique. En effet, l'accès au statut d'adulte handicapé génère également une situation de crise. La spécificité des plaisirs s'ancre dans le dépassement d'un certain nombre de problèmes dont beaucoup semblent communs à tous.

L'atmosphère surprotectrice qui entoure la personne handicapée limite les expériences d'autonomisation. La plupart des personnes handicapées subissent, dans l'enfance ou dans la phase post-traumatique, un «maternage " pendant des périodes plus ou moins longues. Elles vont devoir s'extraire de ce cadre omniprésent constitué des parents, de l'entourage, et des institutions de prise en charge. Les effets certes constructifs, voire indispensables, $\mathrm{du}$ maternage pendant une certaine phase en génèrent ensuite inexorablement d'autres, indésirables : cette surprotection peut amener le sujet à une situation de forte dépendance.

"J'ai été d'un hôpital à un autre, pour réduire les fractures; d'une opération à une

autre, on n'a fait que ça ». (0)

Qu'il s'agisse du deuil du corps de l'enfant pour les uns (valides et handicapés de naissance) ou du deuil d'un corps intègre pour les autres (handicapés traumatiques), la question de la perte est centrale. En ce sens, l'analyse de la situation des personnes handicapées physiques rejoint la problématique de l'adolescence.

Selon A. Birraux (1990), l'adolescence est la crise du désir ». Elle exprime le deuil de l'enfance et la projection sur un avenir inconnu. C'est le passage existentiel nécessaire d'un désir attaché à la génération précédente à un désir voué à s'adapter ou à se lier ailleurs. A. Birraux note un aspect particulier de l'adolescence qui est l'importance de la mise en avant du corps.

Pour la personne handicapée d'origine traumatique, le deuil sera celui du corps intègre dans lequel elle a grandi et a été aimée (pendant l'enfance mais aussi lors de sa vie adulte). Le corps, totalement transformé, d'un point de vue fonctionnel, mais aussi sensitif, apparaît comme inconnu. Pour les handicapés de naissance ou lors de la petite enfance, la transformation pubertaire ouvre les portes d'un nouveau plaisir, d'un plaisir adulte. Mais pour les uns comme pour les autres, cette «métamorphose corporelle » les installe dans un corps adulte « hors-normes ».

P. Mâle (1982), quant à lui, parle de crise pubertaire qu'il distingue de la crise juvénile désignant l'ensemble des difficultés pouvant surgir de l'évolution psychosociale nécessaire à l'adolescence. Pour cet auteur, la crise pubertaire est liée aux transformations pubertaires nécessitant «une adaptation sous deux aspects principaux : la reconnaissance de son propre corps et l'accès à la vie sexuelle génitale » (Mâle P, 1982). Plus précisément, P. Mâle élabore la notion de dysharmonie pubertaire correspondant à une inadéquation entre le développement somatique et pulsionnel et le développement psychologique. Cette inadéquation peut se produire dans un sens (puberté précoce, avec intrusion d'une nouvelle problématique pulsionnelle dans une organisation libidinale restée infantile) ou dans un autre (puberté retardée avec évolution intellectuelle normale, d'où difficulté d'aboutir à une image de soi valable, refus de la compétition et difficulté de la confrontation avec les autres, fréquence des thèmes d'échec marquant une sorte de « castration vécue, etc.). 

relatifs au plaisir. Mais il est apparu que les personnes handicapées étaient confrontées à un certain nombre d'interdits "surnuméraires ", c'est-à-dire qui viennent s'ajouter à ceux qui peuvent s'appliquer à tout un chacun. Cette deuxième lecture a donc été guidée par la recherche d'ensemble de mots, de phrases ou de paragraphes qui pouvaient rendre compte de ces interdits surnuméraires subjectivement perçus. Certains sont verbalisés comme interdits sociaux alors que d'autres relèvent d'interdits plus personnels.

Les interdits sociaux ou la peur d'affronter le regard de l'autre

«Ah, Parce que l'autre jour j'ai été chez un kiné... J'y vais et puis le type me dit, il avait un de ses clients el il dit au type: «Tiens, le vais le présenter un autre de les collègues à toi, IMC comme toi. ». Je fais. «Mais attendez en société quand vous présentez les gens vous dites soit le prénom soit le nom mais pas le handicap. Je dis «C'est complètement nul ça ». Moi je me suis abstrait depuis 87 du milieu des centres [de rééducation] et de ce genre de conneries donc je ne supporte pas qu'on réduise les gens à ça ». (F)

(A propos d'appareillage aux jambes). «Et ma copine elle, elle en avait des deux jambes, elle me dit, moi quand je mets ça, je coupe tout l'effet a mon mari, alors j'en ai parlé à ce docteur je lui ai dit « vous voyez j'ai une amie, el bien son mari lui a dit ça. ». Et elle, elle dit : « Quand c'est comme ça on fait chambre à part. ». Allez dire ça à une jeune femme qui n'a même pas quarante ans, mais c'est dingue!! Vous voyez donc, ne serait-ce qu'un docteur qui pourtant est spécialiste des problèmes physiques et qui dit «Ben si vous avez des problèmes avec votre conjoint faîtes chambre à part ». C'est dingue! Vous voyez (...). On ne conçoit pas un handicapé, le plaisir et un handicapé ça ne va pas ensemble. ». (E) nombre d'interdits relatifs au statut social.

Pour certaines personnes le fait d'être handicapé renvoie nécessairement à une absence de reconnaissance, l'impression de ne pas être considéré en personne adulte, 
l'impossibilité d'être un individu respectable, d'être autre chose qu'un handicapé. Cette dimension peut être illustrée par la gêne face à des tutoiements intempestifs, l'impression de n'attirer que des personnes handicapées, ou des valides mal dans leur peau. La personne handicapée est vécue par le reste de la société comme faisant partie d'un ensemble homogène qui possède des valeurs ou des caractéristiques communes à tous, ce qui fait dire à l'un d'entre eux : « un handicap, c'est un état physique, c'est pas un statut social».

Les interdits relatifs à la sexualité renvoient, là encore, à la difficulté qu'éprouvent les personnes handicapées de se sentir intégrées dans la société « ordinaire ».

La vie en collectivité durant toute l'enfance et l'adolescence n'est pas non plus un facteur contribuant à un épanouissement sexuel puisqu'elle empêche l'intimité et peut favoriser paradoxalement des pratiques dans lesquelles l'individu ne se sent pas toujours respecté.

Enfin, les attitudes d'hostilité des personnes valides de l'entourage (famille, personnel médical) sont parfois douloureuses et conduisent à penser que la société dans son ensemble n'est pas prête à concevoir l'activité sexuelle comme allant de soi chez les personnes handicapées.

Le malaise de se trouver au milieu d'autres personnes qui risquent de vous regarder fait référence à l'interdiction d'exposer son corps devant les autres (à la piscine ou à la plage par exemple). Les interdits de parole, liés à une certaine pudeur, portent par exemple sur les problèmes urinaires ou de selles. Alors qu'ils sont prégnants chez de nombreuses personnes en fauteuils, aborder ces problèmes avec l'entourage nécessite d'être en très grande confiance pour assumer en collectivité les contraintes qu'ils impliquent (obligation de se sonder régulièrement, donc de s'absenter par exemple au milieu d'un repas...). Ils constituent des limites importantes pour les sujets handicapés.

Enfin, l'interdit social peut se traduire clans le fait de ne pas s'autoriser la relation sociale elle même: la gêne causée à l'entourage lorsqu'il regarde la personne handicapée, le fait d'être interpellé plus rarement par les valides semblent opérer une limite à la relation sociale que la personne handicapée n'est pas toujours prête à franchir parce qu'elle a l'impression de choquer » les gens qui sont autour d'elle.

Tous ces interdits renvoient à la perception qu'ont les personnes handicapées des représentations sociales que la majorité des "valides » entretiennent à leur égard. A partir de cette analyse, nous pourrions avancer l'idée qu'une personne handicapée physique a le sentiment, dans ses relations avec le social d'être vécue a priori comme peu respectable, asexuée, dépendante, présentant une réalité physique gênante, que l'on ne regarde pas, que l'on évite de préférence et dont on ne perçoit rien d'autre que son statut de handicapé.

41 A cette dimension de limite sociale, s'ajoutent toute une série de barrières, qui semblent provenir de la personne handicapée elle-même, qui nous ont paru plus autocentrées, moins dépendantes de l'entourage valide mais néanmoins jouant un rôle fondamental dans la question de l'accès au plaisir.

Les interdits personnels ou la difficulté de s'accepter

Cette seconde catégorie d'interdits renvoie à des comportements d'auto-limitation des sujets par rapport à des situations perçues comme trop pénibles.

43 Les limites fonctionnelles génèrent des interdits. Certains déplacements seront évités (escaliers, chemins ruraux, transport en communs...) car ils sont perçus comme trop 
contraignants. Même si des palliatifs peuvent être envisagés (se faire porter), cette éventualité reste difficile à accepter pour le sujet. Il en va de même pour certains actes de la vie quotidienne comme se faire conduire, se faire servir...

La sexualité pâtit également de ces atteintes fonctionnelles, qui peuvent limiter la possibilité de caresser pour un tétraplégique par exemple. On notera que les discours varient selon que l'atteinte est fonctionnelle et/ou sensitive. Ainsi un homme pour qui l'éjaculation est impossible nous exprime son malaise devant sa difficulté «à bien faire l'acte».

Mais la sexualité renvoie également à d'autres difficultés d'ordre relationnel. Comment rencontrer un partenaire, alors qu'on a l'impression qu'on ne peut pas plaire, qu'on ne sera pas capable de répondre à l'attente de l'autre. De plus, ces personnes ont souvent le sentiment, expérience vécue pour certains, que les personnes valides recherchant le contact sont mues par le désir « d'essayer avec une personne en fauteuil » et non par une réelle reconnaissance d'eux-mêmes. L'impression de ne pas être désirable, de ne pas pouvoir faire face est souvent compensée par la recherche ou l'attente du partenaire idéal avec qui il serait possible de vivre le " grand amour »...

L'état physique général du sujet est un troisième facteur d'auto-limitation. Les problèmes médicaux, les douleurs, la fatigue physique font généralement partie de la vie quotidienne, ce qui contribue également à réduire l'activité, fatigabilité supérieure à la moyenne, douleurs lombaires qui obligent à rester couché, prise de poids qui limite très rapidement l'aisance dans les déplacements en fauteuil, douleurs des bras après un déplacement trop important sont autant d'exemples de réalités qui restreignent l'activité bien au-delà de la seule impossibilité de marcher.

"Quand j'ai mal nulle part, il y a quand même des périodes, c'est excellent au niveau de la vie quoi. Je peux mener à bien une journée de fac, faire un entraînement le soir, et après faire, une bouffe chez des copines, sans problème. Tandis que si j'ai mal au dos, je vais faire une des activités, et puis il va falloir que je m'allonge. ». (C)

47 La perception de ces limites physiques contribue à l'émergence d'interdits corporels qui visent à préserver un "capital santé ». Ces personnes évitent de s'abîmer physiquement en s'aménageant des temps de récupération et en limitant l'intensité des activités de leur vie quotidienne.

Le cumul de ces interdits aboutit à l'impossibilité pour les personnes handicapées d'avoir accès à l'aventure, à l'imprévu : toute activité doit être anticipée et planifiée : «Le lieu comporte-t-il des escaliers, des toilettes accessibles, le voyage ne sera-t-il pas trop fatiguant, comment vont m'accueillir les gens?» illustrent les «calculs» continuels que la personne en fauteuil est contrainte d'effectuer et qui peuvent l'amener à renoncer, lorsque le projet présente un rapport coût-bénéfice négatif.

"Mais d'abord, c'est ces contraintes avant qui font qu'il faut réfléchir deux fois avant d'y aller et je pense que le plaisir il est sans réfléchir C'est on m'appelle : pouf «Allez tu viens?». Allez on part. On calcule quoi ? Il n'y a rien à calculer. C'est là où... Les meilleurs moments sont là, où on calcule rien, Là, en fauteuil il faut tout calculer Donc le plaisir est un peu diminué je dirais. ». (A)

49 Par ailleurs cette nécessaire planification des actes quotidiens est souvent vécue comme une frustration importante et « diminue le plaisir »

Plaisir du dépassement de l'interdit 
50 A partir de ces interdits surnuméraires, qui limitent nécessairement la personne handicapée, ou qui lui donnent le sentiment d'être une personne limitée, se développe paradoxalement une série de plaisirs afférents qui trouvent place dans le dépassement de l'interdit. Si ce n'est pas l'unique source de plaisir, elle en est une qui tient une large place dans la vie de ces personnes. Ces types de plaisirs s'organisent tous autour de l'intention et de la réalisation de projets qui permettent de quitter le statut de handicapé, d'accéder à la norme, de manière provisoire ou non : ils procèdent de ce que l'une des personnes interrogées résumait en disant qu'elle « gagnait en validité ».

Au-delà des limites sociales

«Par exemple quand il y a des soirées, si je n'ai pas bu un petit coup, je ne vais pas danser. J'ai du mal a le concevoir. Il y en a qui le font, moi je le fais aussi mais il faut d'abord que je boive un peul coup avant pour enlever les barrières. Après oui après j'y vais $(\mathrm{H})$

51 La possibilité de la rencontre, de provoquer la discussion avec une personne inconnue, le contact avec les autres, font tomber les barrières qui existaient et provoquent du plaisir. Accéder a ce plaisir nécessite parfois l'utilisation de moyens détournés comme boire un petit coup pour oser la rencontre avec des valides ou encore séjourner en centre spécialisé, où la rencontre des autres est plus facile d'accès puisqu'il s'agit de pairs (autres personnes handicapées).

«Moi j'obtiens vraiment du plaisir dans les rapports sexuels mais pas... Evidemment, ça n'a rien à voir avec ce que j'avais avant, parce qu'on développe une sensibilité, ça dépend des gens, moi j'ai un côté vachement... sensuel. Je fais de la sculpture, je fais du dessin, je suis très sensuel donc je reporte, j'ai pas mal de facilités à reporter ce que j'ai perdu... Parce qu'en discutant avec la sexologue, elle me dit - « Il ne faut pas faire l'amour pour faire plaisir à l'autre ». Je lui dis : « Mais si, niais dites vous bien que quand ma partenaire prend du plaisir, je prends mon pied!"

«J'ai développé des sensibilités autres qui m'épanouissent différemment, surtout que j'allais dire autant, je dirais même mieux, parce que je pense que je comprends mieux le plaisir féminin qu'avant... » . (G)

52 L'accès à la sexualité, nous l'avons vu, pose un problème majeur : les personnes qui parviennent à trouver du plaisir dans ce domaine expliquent comment elles ont dépassé les limites auxquelles elles étaient confrontées. Ainsi, les personnes interrogées parlent d'un changement de représentation qui a dû s'opérer et qui leur permet maintenant de s'épanouir sexuellement. Profiter de la sexualité signifie avant tout avoir rompu avec le registre de la prouesse physique et avoir accepté de rechercher de nouvelles sensations, de découvrir de «nouvelles zones érogènes ", qui apportent du plaisir.

Le plaisir de faire un enfant est lui aussi abordé et l'unique personne de notre échantillon qui a eu un enfant nous dit son bonheur d'y être parvenu. Ce plaisir " ordinaire » est encore renforcé par un plaisir dans le dépassement des résistances sociales qu'il constitue : prouver qu'on peut procréer, qu'on peut élever un enfant, qu'on peut être parent. Le thème des enfants souhaités est revenu plusieurs fois dans les entretiens.

«Ca veut dire que moi je voulais prouver qu'un handicapé physique peut procréer en fait, et je l'ai eu, et je l'ai éduqué avec mes difficultés financières et puis assez seule puisque mon couple n'a pas tenu, mais pas du tout pour des problèmes de santé, non pas du tout... Oui pour moi c'est un défi... ». (E) 

comportements et/ou des statuts, qui sont a priori réservés aux personnes valides où que l'entourage proche considère comme tels. Par exemple, le plaisir de passer le permis de conduire et de prendre sa voiture seule s'articule sur le dépassement des interdits d'indépendance, de déplacement. Le fait d'accéder à des résultats sportifs, ou de faire partie d'une élite sportive va dans le même sens. Celui d'être mère (ou père) de famille également.

« Donc je pense que quelque part j'avais envie de lui prouver qu'il se plantait, une fois de, plus [son père], et que je pouvais faire un tas de choses comme les gens valides. ( ... ), donc au niveau du permis de conduire aussi, des études pareil, donc là c'était une revanche, le bac, pareil, et le championnat de France pareil ». (c)

Au-delà des limites personnelles

(A propos du plaisir de la nature)

«En fait depuis que j'ai le fauteuil électrique, je peux le faire [promenades à l'extérieur]. Tant que je n'ai pas eu le fauteuil électrique je ne pouvais pas le faire et je n'en éprouvais pas le besoin. A partir du moment où je pouvais le faire, je l'ai toujours fait. ». (D)

De la même manière, le dépassement des limites que nous avons considérées ici comme plus autocentrées procure également un grand plaisir. Dépasser ses limites physiques en prenant des cours de natation, s'obliger à sortir pour voir du monde, garder une hygiène de vie pour optimiser son rendement physique permettent souvent de parvenir à des résultats qu'on ne soupçonnait pas pouvoir atteindre auparavant.

Dépasser ses limites personnelles signifie également agir pour diminuer les contraintes matérielles ou autre, en achetant un fauteuil plus performant, se procurant des lentilles de contact pour remplacer des lunettes très handicapantes... etc.

Ce dépassement peut entraîner également des choix de vie important, comme celui de travailler plutôt que de vivre d'allocations ou de décider d'avoir des enfants.

Troisième lecture : le sport : passeport au plaisir?

Le sport a été un des thèmes fondamentaux du travail présenté ici. Tous les entretiens ont été volontairement orientés sur cette question. En ce qui concerne la question du plaisir, les personnes interrogées qui pratiquaient une (des) activité(s) sportive(s) ont pu apporter des éléments intéressants qui nous conduisent à penser que le sport est un vecteur du plaisir, un « passeport » au plaisir.

«Le plaisir essentiel que je retire dans ma vie de handicapée vient essentiellement

du sport. Euh, ça donne avec le handicap, une autre façon de voir les choses. ». (C)

Le sport est un mobilisateur. A ce titre, il peut donner du sens à l'existence. Nous avons donc effectué une troisième lecture des entretiens, en ne conservant que les discours des personnes qui pratiquaient une activité sportive régulière. Comme précédemment, nous avons fait une analyse thématique en isolant tous les groupes de mots ou de phrases se rapportant au plaisir, que nous avons ensuite classés en plusieurs catégories.

« A la limite de l'épuisement disons et quand on s'arrête, c'est là où on est bien quoi. ». (B)

Plusieurs personnes soulignent la place centrale qu'occupe le sport dans leur vie, ainsi que les transformations importantes de leur vie quotidienne occasionnées par la pratique sportive. Le sport est associé à des plaisirs multiformes dont la caractéristique commune est l'ouverture à d'autres plaisirs, dépassant le cadre sportif.

«J'étais bien quand je n'en pouvais plus quoi. Quand il fallait il que je me couche, là,

j'étais heureuse, Quand je transpirais, ça ne m'était jamais arrivé par exemple. 
C'était débile, mais le fait d'être essoufflée, un truc que je n'avais jamais connu: être essoufflée après un match de Basket. Avoir mal partout mais là, le savais pourquoi j'avais mal. Pas parce que j'avais mal au dos à cause de mon handicap, mais parce que j'étais, au même litre qu'un sportif crevée par l'effort. ». (C)

61 Le sport est une dépense d'énergie. C'est elle qui procure du plaisir. Se défouler, faire un exercice à la limite de l'épuisement, envie de se "défoncer", se "crever ", se donner à fond sont autant de moyens de se sentir bien, d'éprouver du plaisir par l'éprouvé du corps. Les entretiens sont unanimes sur ce point: le sport procure du plaisir parce qu'il y a une notion d'effort, de dépense physique qui, une fois terminée, provoque du bien-être. C'est l'éprouvé du corps en action mais aussi de sensations provoquées par l'effort sportif et consécutives à celui-ci qui donnent du plaisir.

Le sport est également le moyen de développer de nouvelles possibilités insoupçonnées jusqu'alors. Autant sur le plan physique que social, il permet de réussir quelque chose que les personnes interrogées ne pensaient pas pouvoir réussir. Au-delà du plaisir immédiat de la pratique sportive, celle-ci conduit la personne handicapée à franchir des portes a priori fermées : s'autoriser à sortir seul pour aller s'entraîner, à rencontrer d'autres sportifs, à partir plusieurs jours dans le cadre d'un déplacement.

Dans cette perspective, le sport apparaît comme un excellent vecteur de dépassement de l'interdit.

"Je ne pouvais même pas essayer de me muscler, et je n'arrivais même pas à l'époque à mettre mes chaussures, ou des choses comme ça quoi. C'est en ça que le sport m'a fait gagner après : à partir du moment où j'ai su que j'étais consolidée. J'ai commencé à faire un petit peu des exercices, apprendre à me mettre par terre et à me relever, même si c'est pas utile tous les jours. (...) J'ai énormément gagné depuis, je ne me casse pas, je me suis musclée. J'ai fait des pompes : on prend conscience de son corps. ». (C)

64 Le sport peut être également tout simplement un moyen de se maintenir en bonne santé, ou du moins contribuer à un bien-être général parce qu'il fait perdre du poids ce qui facilite les déplacements en fauteuil, parce qu'il muscle le dos et permet un meilleur redressement, etc.

» Moi, il y a une relation immédiate entre ma pratique sportive, mon niveau de moral et mon état physique général. Ce sont trois éléments : quand je fais du sport le moral est en hausse et mon physique c'est sans commune mesure. En quinze jours de temps je passe d'un état à un autre quoi, je peux gagner. En maintien dorsal, je peux gagner presque 5 à $10 \mathrm{~cm}$ de hauteur parce que mon corps s'est redressé. ». (F)

Les activités physiques, notamment aquatiques mais aussi de glisse ou autres sont le moyen d'avoir des sensations qui procurent du plaisir : vitesse, glissement dans l'eau, absence de contrainte physique en plongée, mais aussi domination de la peur en équitation proposent un moyen de vivre des situations grisantes, d'expérimenter des sensations jusqu'ici inconnues et non exemptes d'une dimension de plaisir.

« Le cheval, j'ai peur, j'ai tout le temps peur donc c'est peut-être ça aussi, j'ai besoin

d'avoir peur et j'aime. », (D)

De même, réussir un «beau geste ", marquer un «beau point ", effectuer « une belle technique » sont des actions très recherchées qui provoquent immanquablement un grand plaisir lorsqu'elles sont réalisées.

« Si, de la plongée j'en ai fait, je me suis régalé. Mais bon, parce que là, au contraire, quand on est sous l'eau, il n'y a plus de handicap. On peut aller où on veut, on se déplace comme on veut. ». (A) 
«Il y a le ski, le side-car, ça je pense que je le referai, (...). Des ballades sans

habitacle autour. C'est bas près du sol, ça fait des sensations. ». (H)

ne paraîtront pas spécifiques à cette population mais n'est-ce pas justement parce que le sport leur permet l'accès à des plaisirs "communs"? Le sport se présente donc comme " passeport » au plaisir dans la mesure où il s'inscrit dans le dépassement des interdits par rapport à soi et aux autres...

«On a fait les premiers stages en équipe de France, ( ... ). Et là c'était intéressant parce qu'on se sentait vraiment athlète. (...). Donc c'est vrai que bon c'était quelque part un peu sympa de se dire qu'on faisait partie d'une certaine élite quoi, même si ça n'a plus aucun rapport avec ce qui se passe maintenant... ». (p)

«Quand on est en haut de tableau, en demi-finale il y a l'envie de gagner qui est là, il y a tout le monde qui nous regarde, c'est pas négligeable. Là, on va puiser les réserves, c'est là où c'est bon. ». (A)

«Plaisir de se faire mal pour pouvoir après gagner là le but c'est de gagner, d'être le meilleur, c'est ça mon objectif, ». (A)

«Mes premières grosses joies, ça a été le plus beau jour de ma vie quand j'ai fini deuxième au championnat de France. Là ça a été un plaisir. C'était fou quoi, là je ne touchais plus terre. ». (C)

L'accès au plaisir : une condition de socialisation?

70 Ainsi, les entretiens révèlent chez les personnes handicapées des plaisirs ordinaires, plaisirs communs qui correspondraient aux représentations les plus communes qu'on se fait du plaisir. Mais à côté de ces plaisirs courants apparaîtraient des "plaisirs clandestins». Ils fonctionnent dans le quotidien à l'insu de tous. Ils ne sont pas nommés par leurs vrais noms. Et cependant ils sont organisés, codés, et mis en réseaux pour survivre et prospérer.

71 Mais l'important est moins de les débusquer et de les identifier, que de reconnaître en eux, chez les personnes handicapées, les gages d'une «institution du plaisir » qui dépasserait largement les déterminants sociaux pour toucher l'essence même du social : il ne saurait y avoir de société où le plaisir en tant que tel ne serait institué, et instituant.

72 Et si de l'institution à la normalisation il n'y a qu'un pas, C'est un pas que les personnes handicapées ne tardent pas à franchir, car en voulant "gagner en validité », elles gagnent aussi en plaisir, et par là doublement en normalité.

73 Mais la trajectoire qui les y conduit mérite un détour, car on n'accède pas à la normalité, même par le plaisir, d'emblée: il faudrait justement pouvoir faire l'expérience du plaisir, ce qui, dans les représentations communes, est interdit aux handicapés. Et au-delà de l'interdit, l'injonction de «double contrainte » présente même un caractère quelque peu pervers et schizophrénique: «Tu n'as pas droit au plaisir, mais tu dois t'intégrer!». Or il semblerait bien que le plaisir ne soit pas étranger, comme support de communication, à l'instauration d'un langage commun et 
de représentations communes. Comment donc adhérer à une communauté quand l'accès à son plaisir est interdit? Et inversement, comment accéder au plaisir en dehors d'une communauté, en dehors de l'intersubjectivité de l'échange implicite de sens ?

Pour les personnes handicapées, comme probablement pour la plupart des communautés restreintes, une solution s'imposerait alors: prendre du plaisir entre pairs, du ou des plaisirs interdits, des plaisirs clandestins. Paradoxalement, ce serait cette expérience clandestine de l'échange intersubjectif de sens, par plaisir interposé, qui permettrait l'échange de sens commun, et par là l'intégration.

Encore faut-il que la personne handicapée passe par l'épreuve du plaisir !

76 A ce sujet, les thèmes relevés laissent apparaitre une progressivité du plaisir, du plaisir sensori-moteur au plaisir symbolique, du plaisir de perception au plaisir conceptuel, du plaisir simple au malin plaisir. Mais si le cours du plaisir se révèle identique dans le monde valide, alors ce serait bien l'expérience du plaisir qui ferait entrer la personne handicapée de plain pied dans le monde de l'Homme. Le problème reste toutefois que ces hommes ne reconnaissent pas aux handicapés le droit au plaisir. Et le pire est que les personnes handicapées ont d'autant mieux intégré l'interdit qu'elles vivent en état de deuil permanent, et que tant que l'on est en deuil, on doit avoir la délicatesse de se dispenser de plaisir.

77 Les entretiens laissent apparaître la profonde intégration de ces interdits "surnuméraires", non seulement chez les personnes handicapées par accident ou maladie, qui sont en deuil de leur corps valide, niais aussi chez les personnes handicapées de naissance, qui n'ont pas fait le deuil d'un corps attribué.

Vers une théorie du plaisir?

$\mathrm{Au}$ terme de cette étude exploratoire, qui visait à mieux comprendre les processus de plaisir chez les personnes handicapées physiques (sportives ou non sportives), il semble indispensable de situer les acquis. Cette ultime réflexion devrait permettre de repérer les perspectives et axes d'études à retenir pour approfondir ce familier si mal connu : le plaisir.

79 Parce que la méthodologie ici choisie reposait sur la technique de l'entretien, nous n'avons donc étudié le plaisir que par le biais des discours sur celui-ci. Nos données de terrain sont constituées par ce que disent les sujets de leur éprouvé de plaisir. En conséquence, il s'agit d'une étude portant sur les représentations spécifiques du plaisir au sein d'une population particulière. Cette recherche s'inscrit donc dans le champ de la psychologie sociale.

Remarquons que l'observation directe des comportements hédonistes chez l'humain est délicate (Haye G., 1995). Sans doute est-ce la raison pour laquelle les expérimentations des biologistes, seuls scientifiques actifs sur ce thème actuellement, ont été effectuées essentiellement sur l'animal. Pour compléter notre approche des phénomènes de plaisir, l'élaboration d'une méthode d'observation de l'humain devrait constituer une prochaine étape.

81 L'enjeu théorique du choix de la population ici étudiée réside dans la relation cachée, voire niée, que les handicapés entretiennent avec le plaisir. A l'instar des premières études sur l'intelligence qui se sont effectuées auprès de déficients mentaux, cette étude s'appuie sur un public que l'on peut considérer comme "limité » dans son accès au plaisir. Cette option a permis ici d'identifier un des processus de plaisir: le dépassement de l'interdit ou de la place assignée. 
Elle a permis également de mettre en évidence que l'accès aux plaisirs communs ouvrait la voie à l'intégration sociale. Au moment où, le malaise dans la civilisation » mue le plaisir en valeur (Rauch A., 1986), il n'est pas étonnant qu'il devienne objet et enjeu de connaissance.

\section{BIBLIOGRAPHIE}

Birraux, A. (1990). L'adolescent face à son corps, Paris, Editions Universitaires.

Bon, C. (1995). « L'apport de la phénoménologie dans l'évaluation des situations de handicap », in Ravaud \& Fardeau, Insertion sociale des personnes handicapées. Méthodologie d'évaluation, Paris, Ed.

CTNERHI.

Have, G. (1995). « Introduction à l'étude du plaisir des pratiquants d'APS », Corps et Culture, 1, 45-55.

Mâle, P. (1982). La crise juvénile, Paris, Payot.

Rauch, A. (1986). « Le loisir ou les didactiques du plaisir », STAPS, 7, 13, 23-26.

\section{RÉSUMÉS}

Associer plaisir et handicap physique, malgré l'apparente contradiction de ces deux termes, a été le point de départ de cette recherche. Dans une perspective exploratoire, nous avons menés des entretiens semi-directifs auprès d'adultes présentant un handicap physique, certains étant des sportifs, d'autres non. Les résultats mettent en évidence l'existence tic, plaisirs spécifiques chez ce public, plaisirs qui reposent sur le dépassement d'interdits sociaux ou personnels. Cette étude a, en outre, souligné la fonction du sport comme « passeport » au plaisir pour ce public, dans la mesure où il se constitue en espace de dépassement des limites et où il permet également l'accès à une communauté de plaisir avec les «valides ». Il représente alors une possibilité d'entrer dans un processus qui a pour but de "gagner en validité», situation qui génère un plaisir particulier pour ces personnes,

Associating pleasure and physical deficiency has been the start point of this research, despite the apparent contradiction between these two terms. In an exploratory perspective, we've led semidirective interviews next Io physically disabled adults, some of them practising sport, others don't. Results display the existence of specific pleasures among ibis group, pleasures which are associated with the surpassing of social or personnel interdictions. Furthermore, the study underlines a specific function of sport practise as "passport» to pleasure for these persons, insofar as sport practise is a situation of limits overstepping and because it allows an access to a community of pleasure with «the valids ». Sport is pointing out to this group a Possibility of entering in a specific processus leading to "win in validity». This situation is engendering a specific pleasure among physically disabled people. 
INDEX

Keywords : sport, pleasure, physically disabled people, interdictions surpassing

Mots-clés : plaisir, personnes handicapées physiques, depassement des interdits

\section{AUTEURS}

\section{JEAN-PHILIPPE TURPIN}

Faculté des Sciences du Sport et de l'Education Physique. Laboratoire «Sport, Santé et Développement », UFR STAPS Montpellier.

\section{JEAN-MARC BARBIN}

Faculté des Sciences du Sport et de l'Education Physique. Laboratoire « Sport, Santé et Développement », UFR STAPS Montpellier.

\section{GILLES BUI-XUÂN}

Faculté des Sciences du Sport et de l'Education Physique. Laboratoire «Sport, Santé et Développement », UFR STAPS Montpellier.

\section{ANNE MARCELLINI}

Faculté des Sciences du Sport et de l'Education Physique. Laboratoire « Sport, Santé et Développement », UFR STAPS Montpellier.

\section{JACQUES MIKULOVIC}

Faculté des Sciences du Sport et de l'Education Physique. Laboratoire « Sport, Santé et Développement », UFR STAPS Montpellier.

\section{GRÉGORY NINOT}

Faculté des Sciences du Sport et de l'Education Physique. Laboratoire « Sport, Santé et Développement », UFR STAPS Montpellier.

\section{GUY HAYE}

Equipe « Corps et Culture », UFR STAPS Montpellier. 